\title{
Infective Endocarditis Caused by Extended- Spectrum Beta-Lactamase-Producing Escherichia coli: A Case Report
}

\author{
Tsuneaki Kenzaka $\mathbb{D}^{1,2}$ \\ Yuto Shinkura' \\ Shizuo Kayama ${ }^{3-5}$ \\ Liansheng $\mathrm{Yu}^{3-5}$ \\ Sayoko Kawakami ${ }^{3}$ \\ Motoyuki Sugai ${ }^{3-5}$ \\ Satoru Kawasaki ${ }^{1}$
}

'Department of Internal Medicine, Hyogo Prefectural Tamba Medical Center, Tanba, Japan; ${ }^{2}$ Division of Community Medicine and Career Development, Kobe University Graduate School of Medicine, Kobe, Japan; ${ }^{3}$ Antimicrobial Resistance Research Center, National Institute of Infectious Diseases, Tokyo, Japan; ${ }^{4}$ Department of Antimicrobial Resistance, Graduate School of Biomedical and Health Sciences, Hiroshima University, Hiroshima, Japan; ${ }^{5}$ Project Research Center for Nosocomial Infectious Diseases, Hiroshima University, Hiroshima, Japan
Correspondence: Tsuneaki Kenzaka Division of Community Medicine and Career Development, Kobe University Graduate School of Medicine, 2-I-5, Aratacho, Hyogo-ku, Kobe, Hyogo, 652-0032. Japan

Tel +8I-78-382-6732

Fax +8I-78-382-6283

Email smile.kenzaka@jichi.ac.jp
Introduction: Extended-spectrum $\beta$-lactamase-producing Escherichia coli (ESBL-EC) reportedly accounts for $>20 \%$ of $E$. coli infections and $2.0 \%$ of infective endocarditis cases. Nonetheless, there is a global paucity of reports on infective endocarditis caused by ESBL-EC. Case: An 83-year-old Japanese man who underwent mitral annuloplasty for mitral valve prolapse 5 years ago developed a fever of $38.5^{\circ} \mathrm{C}$. The patient was hospitalized the first time for pyelonephritis and bacteremia due to ESBL-EC and treated successfully with the antimicrobial meropenem for 14 days. Two days after discharge, however, the patient was re-admitted with bacteremia due to ESBL-EC. He was treated successfully with the antimicrobial cefmetazole for 14 days. The patient was admitted to our institution for a third time due to bacteremia again, a day after discharge following meropenem antibiotic therapy. Transesophageal echocardiography showed vegetation in the anterior mitral valve annulus. Magnetic resonance imaging of the head showed septic cerebral embolism. The patient was diagnosed with infective endocarditis due to ESBL-EC and underwent mitral valve replacement. After 6 weeks of antibiotic therapy with meropenem and tobramycin, the patient recovered completely. The causative E. coli strain MS6396 was identified as the E. coli clone ST131 by multilocus sequence typing and confirmed the presence of $b l a_{\mathrm{CTX}-\mathrm{M}-27}$ ESBL gene.

Conclusion: Only six cases of infective endocarditis associated with ESBL-EC have been reported in the past. Moreover, this is the first report of a patient with infective endocarditis bacteriologically or genetically analyzed for ESBL-EC. In future, factors that may cause infective endocarditis in ESBL-EC infections may be clarified through more thorough bacteriological/genetic analyses of ESBL-EC.

Keywords: $b l a_{\mathrm{CTX}-\mathrm{M}-27}$ ESBL, ST131, E. coli, meropenem, infective endocarditis, bacteremia

\section{Introduction}

Extended-spectrum $\beta$-lactamases (ESBLs) can hydrolyze oxyimino-cephalosporins such as cefotaxime, and ceftriaxone. ${ }^{1}$ This has a significant impact on the treatment of Enterobacteriaceae, including Escherichia coli. In recent years, the prevalence of ESBL-producing $E$. coli (ESBL-EC) has increased dramatically worldwide. $^{2,3}$ In Japan, ESBL-EC reportedly accounts for $>20 \%$ of E. coli infections. 4

The incidence of infective endocarditis in Japan is unknown; however, there have been 513 reports in 3 years at 114 hospitals with cardiovascular specialists, and the actual number is considered to be much higher. ${ }^{5} E$. coli has been reported to 
be the causative agent, accounting for $2.0 \%$ of infective endocarditis cases, ${ }^{5}$ even though it is rarely responsible for infective endocarditis, and ESBL-EC is an even rarer causative agent. ${ }^{6,7}$ In this case report, we present the case of a patient with infective endocarditis caused by ESBL-EC.

\section{Case}

An 83-year-old Japanese man presented at our institution with a fever of $38.5^{\circ} \mathrm{C}$ in the first episode. Five years previously, the patient had undergone mitral annuloplasty for mitral valve prolapse. The patient had been taking pravastatin $10 \mathrm{mg} /$ day, rabeprazole $10 \mathrm{mg}$ /day, carvedilol $2.5 \mathrm{mg} /$ day, cibenzoline $150 \mathrm{mg} /$ day, and verapamil 120 $\mathrm{mg} /$ day continuously following the mitral valve surgery. The patient did not smoke or consume alcohol and did not have diabetes mellitus. In addition to the fever, the patient presented with right lower back pain and was examined at our institution. ESBL-EC was detected in two sets of blood cultures and a urine culture. The patient was admitted to the hospital for the first time. We administered antibacterial therapy with meropenem ( $1 \mathrm{~g}$ every 8 hours) to the patient for 14 days for pyelonephritis and bacteremia caused by ESBL-EC. Negative blood cultures were obtained 3 days after treatment initiation. The patient was discharged one day after the completion of antibiotic therapy.

Two days after discharge, he presented with a fever of $38.0^{\circ} \mathrm{C}$ and was admitted for a second time at our institution. As before, ESBL-EC was observed in two sets of blood cultures; however, the urine culture were negative. We initiated antibiotic therapy with cefmetazole ( $1 \mathrm{~g}$ every 8 hours) for 14 days. Three days after treatment initiation, the patient's blood cultures were negative, and he was discharged in good general condition.

However, malaise was observed the day after discharge, and the patient had a fever $\left(37.4^{\circ} \mathrm{C}\right)$ and was admitted for a third time at our institution. His consciousness was lucid and his vitals were as follows: body temperature, $37.4^{\circ} \mathrm{C}$; blood pressure, $100 / 53$ $\mathrm{mmHg}$; pulse rate, 77 beats/minute; respiratory rate, 18 breaths/minute, regular; $\mathrm{SpO} 2,96 \%$ (room air). No conjunctival hemorrhage, nail splinter hemorrhage, Janeway lesions, or Osler's nodules were observed. Heart sounds were regular, and no murmurs were audible.

Blood test results showed white blood cells at 16,000/ $\mu \mathrm{L}$, neutrophils at $90.0 \%$, and C-reactive protein at $8.9 \mathrm{mg} /$ dL. Procalcitonin was $20.3 \mathrm{ng} / \mathrm{mL}$. Two sets of blood cultures yielded ESBL-EC. Antimicrobial susceptibility results are shown in Table 1. The susceptibility results for ESBL-EC detected by blood cultures in the two previous admissions and urine cultures in the first admission were similar. ESBL-EC (designated MS6396) was susceptible to meropenem and cefmetazole.

For repeated bacteremia due to ESBL-EC, the presence of abscess or infective endocarditis was suspected, and the following tests were performed. Computed tomography of the chest, abdomen, and pelvic regions revealed no remarkable findings. Transthoracic echocardiography did not show vegetation, whereas transesophageal echocardiography showed mobile vegetation, $25 \mathrm{~mm}$ in size, in the anterior mitral valve annulus (Figure 1). No valve destruction was noted. Magnetic resonance imaging of the head (Figure 2) and diffusion-weighted and fluid-attenuated inversion-recovery images showed $2-3 \mathrm{~mm}$ sporadic high-intensity areas, mainly in the parietal lobe, which were considered to be septic cerebral embolisms.

The diagnosis was infective endocarditis of the mitral valve and septic cerebral embolism due to ESBL-EC. We started antibiotic treatment with meropenem at a dose of 1 g every 8 hours on admission. The blood cultures were negative 3 days after treatment initiation. We also administered tobramycin $(1.5 \mathrm{mg} / \mathrm{kg}$ every 8 hours) from day 5 onwards, in addition to meropenem for treating infective endocarditis of the mitral valve due to ESBL-EC. A cardiovascular surgery was planned, and the patient was transferred to another institution to undergo mitral valve replacement on day 13. The pathological findings during the surgery were abscess-like changes, including inflammatory cell infiltration in the mitral valve mixed with bacterial mass.

The patient was treated with meropenem and tobramycin for 6 weeks postoperatively, and he recovered completely. Four years after the surgery, no recurrence has been observed to date.

After the patient's treatment was completed, additional bacteriological and genetic examinations were performed on the causative E. coli (MS6396) isolated from the blood culture samples which was obtained at the first hospitalization. DNA obtained via the cetyltrimethyl ammonium bromide DNA extraction method $^{8}$ was used for DNA sequencing using a MiniSeq next-generation sequencer (Illumina, San Diego, CA, USA). For MiniSeq sequencing, the DNA library was prepared using the Nextera XT Library Prep Kit and Nextera XT Index Kit (Illumina), according to the manufacturer's instructions. A total of 1,130,645 paired- 


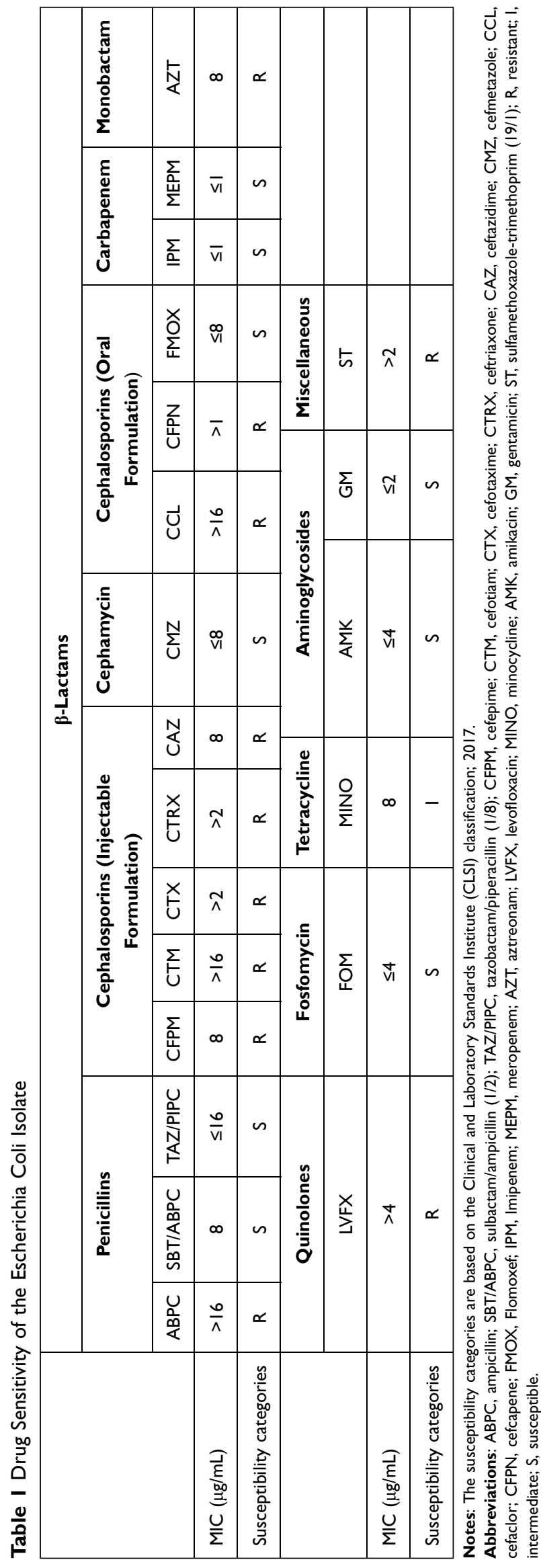

end reads were obtained from the MiniSeq run after adaptor trimming. Trimming of low-quality reads and assembly (average coverage of $56.411 \mathrm{x}$ ) were performed using Shovill v1.0.9 (https://github.com/tseemann/shovill). The MS6396 genome consists of 224 contigs with a length of $5,077,791$ base pairs. The strain was identified as E. coli using KmerFinder $3.1^{9}$ and as the E. coli clone ST131 by multilocus sequence typing (MLST) using MLST 2.0. ${ }^{10} \mathrm{~A}$ search for drug resistant genes using ResFinder-3. $2^{11}$ confirmed the presence of $b l a_{\mathrm{CTX}-\mathrm{M}-27}$ ESBL gene (Table 2). Additionally, the predominant plasmid replicon type was observed to be IncFIA, IncFIB (AP001918), and IncFII (pRSB107) (Table 3). Pathogenesis-related genes were searched using a VFanalyzer, ${ }^{12}$ and we identified the adherence- (pilus or fimbriae), autotransporter-, invasion-, iron uptake-(siderophore), secretion system-, and toxin-related genes (enterotoxin, hemolysin). Details of the pathogenesis-related genes of MS6396 are shown in the Supplemental Table.

\section{Discussion}

Here, we reported the case of a patient who presented at our institution with infective endocarditis caused by ESBL-EC. To the best of our knowledge, infective endocarditis associated with ESBL-EC has been reported in only six cases in the past. ${ }^{6,7,13-16}$ Moreover, bacteriological or genetic analysis of ESBL-EC has not been performed in any of the previously reported cases, making ours the first case to report on ESBL-EC analyses for infective endocarditis.

The E. coli clone ST131 detected in this patient is a common ESBL-EC pandemic clone that has spread globally among human clinical isolates. ${ }^{17}$ E. coli $\mathrm{ST} 131$ is also the most pathogenic drug-resistant $E$. coli clone worldwide. ${ }^{3}$ In a Japanese tertiary hospital, the prevalence of E. coli ST131 among ESBL-EC isolates was 52\% between 2008 and 2011. ${ }^{18}$ Additionally, E. coli strains that produce CTX-M enzymes are globally prevalent in the environment, livestock, and humans, spreading as community-onset diseases and causing urinary and bloodstream infections. ${ }^{19,20}$ In this patient, the onset of pyelonephritis and subsequent bacteremia were observed, and the patient's underlying post-mitral valve surgery status might have led to the infective endocarditis, which required postmitral valve surgery.

Genome sequencing analysis of MS6396 indicated that it belonged to the phylogenetic group B2, which mainly contains virulent extra-intestinal $E$. coli strains $^{21}$ and has 

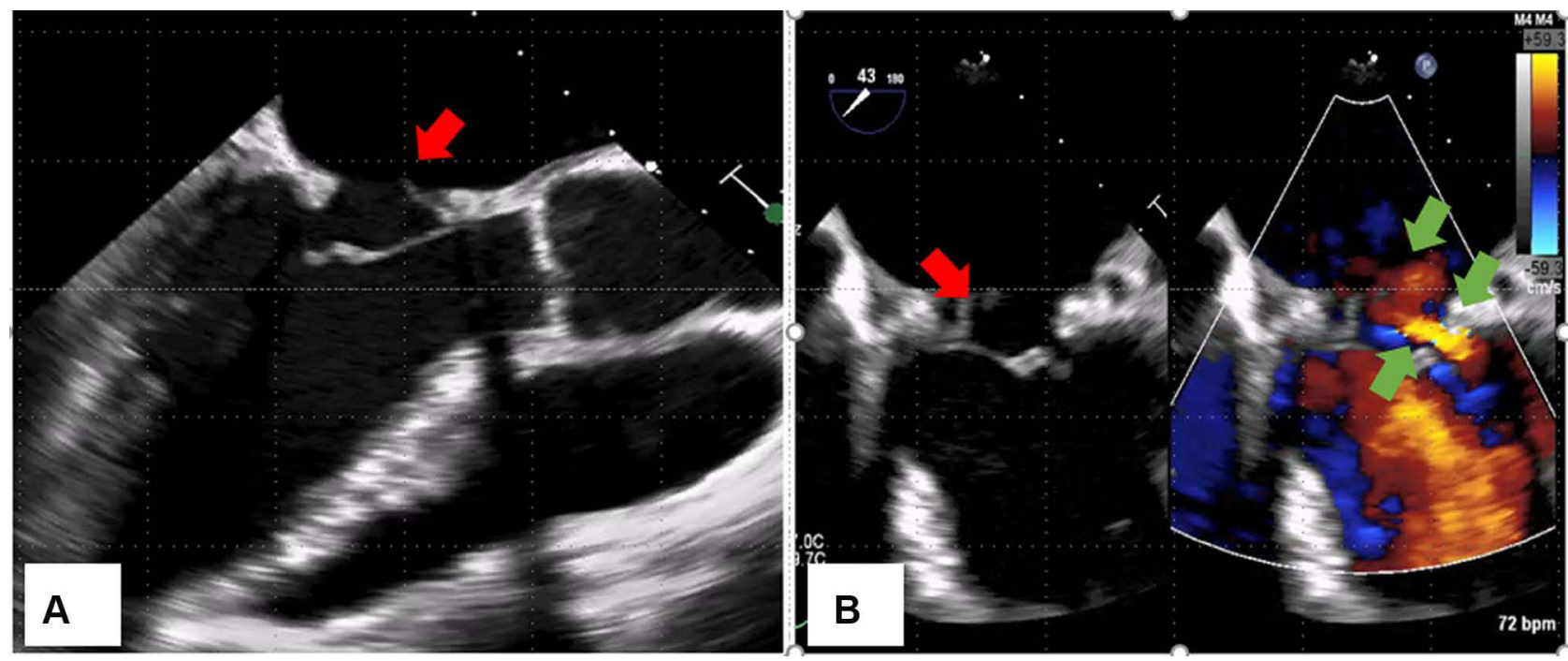

Figure I Transesophageal echocardiography $(\mathbf{A}) 135^{\circ}$, (B) Mitral valve commissure $\left(60^{\circ}\right)$. Mobile vegetation, $25 \mathrm{~mm}$ in size, is observed in the anterior mitral valve annulus (red arrow). Valve destruction is not observed. This is the portion exposed to reverse flow (green arrow).
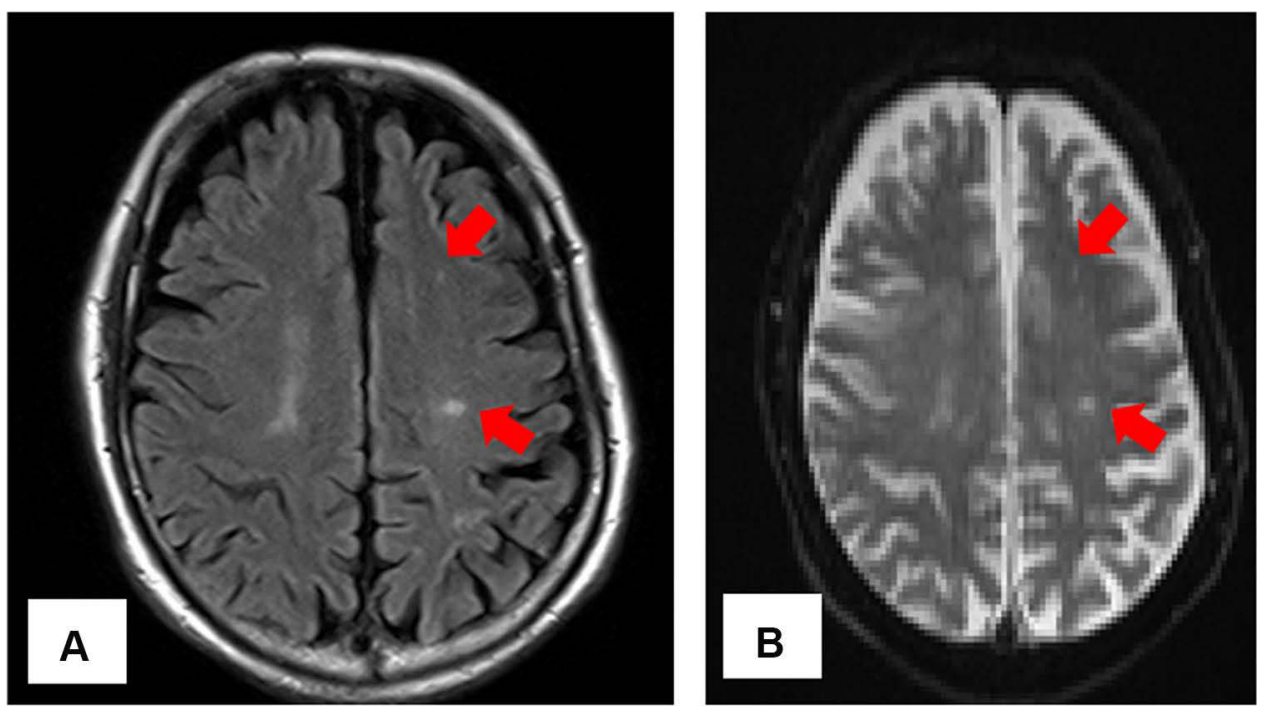

Figure 2 Magnetic resonance imaging of the head (A) fluid-attenuated inversion-recovery images, (B) diffusion-weighted images. Hyperintense areas, mainly in the parietal lobe, are sporadically observed (red arrow) and were considered septic cerebral embolisms.

been implicated in biofilm production. ${ }^{20}$ However, MS6396 did not show biofilm-producing phenotype and lacked the specific or unique genes involved in biofilm production (Supplemental Table). ST131 belonged to the O25:H4 serotype and was reported to be an intestinal adherent-invasive clone. ${ }^{3,22}$ The pathophysiology of MS6396-associated infective endocarditis remains to be elucidated. Further, the virulence of CTX-M-27-producing
E. coli ST131 is not fully understood; however, given their close relationship to CTX-M-15-producing E. coli ST131, which is reportedly highly pathogenic, ${ }^{23}$ it might be assumed that they have a certain pathogenic potential.

In Japan, E. coli accounts for $2.0 \%$ of infective endocarditis cases. ${ }^{5}$ However, ESBL-EC reportedly accounts for $>20 \%$ of E. coli infections in Japan. ${ }^{4}$ Nonetheless, there have been very few reports on infective endocarditis 
Table 2 Drug Resistance Gene Search Results

\begin{tabular}{|l|c|c|c|c|c|c|}
\hline \multicolumn{2}{|l|}{ Beta-Lactam } \\
\hline $\begin{array}{l}\text { Resistance } \\
\text { Gene }\end{array}$ & Identity & $\begin{array}{c}\text { Query/Template } \\
\text { Length }\end{array}$ & Contig & $\begin{array}{c}\text { Position in } \\
\text { Contig }\end{array}$ & $\begin{array}{c}\text { Predicted } \\
\text { Phenotype }\end{array}$ & $\begin{array}{c}\text { Accession } \\
\text { Number }\end{array}$ \\
\hline bla $_{\text {CTX-M-27 }}$ & 100 & $876 / 876$ & contig00I88 & 248.1123 & Beta-lactam resistance & AYI56923 \\
\hline
\end{tabular}

Table 3 Plasmid Search Results. The Following Replicons Were Detected

\begin{tabular}{|l|c|c|l|c|c|c|}
\hline Plasmid & Identity & Query/Template Length & Contig & Position in Contig & Note & Accession Number \\
\hline Coll56 & 98.59 & $142 / 154$ & contig00II5 & 6753.6894 & NC00978I \\
IncFIA & 100 & $388 / 388$ & contig00I20 & 136.523 & AP00I9I8 \\
IncFIB (AP00I9I8) & 97.95 & $682 / 682$ & contig00I09 & 8601.9282 & AP00I9I8 \\
IncFII (PRSBI07) & 100 & $261 / 26 I$ & contig00I56 & 1264.1524 & pRSBI07 & AJ85I089 \\
\hline
\end{tabular}

associated with ESBL-EC worldwide. 6,7,13-16 Collection of patient data on drug-resistance genes, plasmid replicon types, and pathogenesis-related genes may reveal factors that lead to infective endocarditis in ESBL-EC infections.

\section{Conclusion}

This report presents an extremely rare case of a patient with infective endocarditis due to ESBL-EC. In the future, factors that may cause infective endocarditis associated with ESBL-EC might be clarified through more bacteriological/genetic analyses of ESBL-EC.

\section{Data Sharing Statement}

The sequence data for E. coli MS6396 have been deposited in the DDBJ Sequence Read Archive (DRA) under accession number DRA012470.

\section{Consent for Publication}

Written informed consent was obtained from the patient for the publication of this case report and accompanying images. A copy of the written consent is available for review by the Editor of this journal.

\section{Acknowledgments}

We would like to thank Kazunori Yoshida (Department of Cardiovascular Surgery, Nishinomiya Watanabe Cardiovascular Center), who performed surgery on this patient.

\section{Author Contributions}

All authors contributed to data analysis, drafting or revision of the article, have agreed upon the journal to which the article will be submitted, have given final approval to the version to be published, and agree to be accountable for all aspects of the work.

\section{Funding}

There is no funding to report.

\section{Disclosure}

The authors declare that they have no conflicts of interest.

\section{References}

1. Paterson DL, Bonomo RA. Extended-spectrum beta-lactamases: a clinical update. Clin Microbiol Rev. 2005;18:657-686. doi:10.1128/ CMR.18.4.657-686.2005

2. Hara T, Sato T, Horiyama T, Kanazawa S, Yamaguchi T, Maki H. Prevalence and molecular characterization of CTX-M extended-spectrum $\beta$-lactamase-producing Escherichia coli from 2000 to 2010 in Japan. Jpn J Antibiot. 2015;68:75-84.

3. Nicolas-Chanoine MH, Bertrand X, Madec JY. Escherichia coli ST131, an intriguing clonal group. Clin Microbiol Rev. 2014;27:543574. doi:10.1128/CMR.00125-13

4. Miyazaki M, Yamada Y, Matsuo K, et al. Change in the antimicrobial resistance profile of extended-spectrum $\beta$-lactamase-producing Escherichia coli. J Clin Med Res. 2019;11:635-641. doi:10.14740/ jocmr3928

5. Nakatani S, Mitsutake K, Ohara T, et al. Recent picture of infective endocarditis in Japan-lessons from Cardiac Disease Registration (CADRE-IE). Circ J. 2013;77:1558-1564. doi:10.1253/circj.cj-12-1101

6. Fordyce CB, Leather RA, Partlow E, Swiggum EA. Complete heart block associated with tricuspid valve endocarditis due to extended spectrum $\beta$-lactamase-producing Escherichia coli. Can J Cardiol. 2011;27:263-e17. doi:10.1016/j.cjca.2010.12.011

7. Spaleniak S, Romejko-Ciepielewska K, Lubas A, Ryczek R, Niemczyk S. Infective endocarditis in the course of urosepsis E. coli ESBL (+) in a patient with Goodpasture's syndrome. Kardiol Pol. 2015;73:670. doi:10.5603/KP.2015.0154

8. Avgustin G, Wright F, Flint HJ. Genetic diversity and phylogenetic relationships among strains of Prevotella (Bacteroides) ruminicola from the rumen. Int J Syst Bacteriol. 1994;44:246-255. doi:10.1099/ 00207713-44-2-246 
9. Larsen MV, Cosentino S, Lukjancenko O, et al. Benchmarking of methods for genomic taxonomy. J Clin Microbiol. 2014;52:15291539. doi:10.1128/JCM.02981-13

10. Larsen MV, Cosentino S, Rasmussen S, et al. Multilocus sequence typing of total-genome-sequenced bacteria. J Clin Microbiol. 2012;50:1355-1361. doi:10.1128/JCM.06094-11

11. Zankari E, Hasman H, Cosentino S, et al. Identification of acquired antimicrobial resistance genes. $J$ Antimicrob Chemother. 2012;67:2640-2644. doi:10.1093/jac/dks261

12. Liu B, Zheng D, Jin Q, Chen L, Yang J. VFDB 2019: a comparative pathogenomic platform with an interactive web interface. Nucleic Acids Res. 2019;47(D1):D687-D692. doi:10.1093/nar/gky1080

13. Lupse M, Flonta M, Straut M, Usein CR, Tantau M, Serban A. Recurrent Infective endocarditis of the native aortic valve due to ESBL producing Escherichia coli (e coli) after therapeutic ERCP. $J$ Gastrointestin Liver Dis. 2012;21:217-219.

14. Modi HH, Modi SH, Siddiqui BR, Andreoni JM. A rare case of prosthetic valve endocarditis caused by extended-spectrum $\beta$-lactamase producing Escherichia coli. J Glob Infect Dis. 2011;3:99-101. doi:10.4103/0974-777X.77310

15. George S, Varghese J, Chandrasekhar S, et al. Gram-negative bacteria causing infective endocarditis: rare cardiac complication after liver transplantation. World J Hepatol. 2013;5:296-297. doi:10.4254/wjh.v5.i5.296

16. Saboe A, Sari MT, Cool CJ, et al. Cutaneous vasculitis and generalized lymphadenopathy associated with extended-spectrum beta-lactamase (ESBL)-producing Escherichia coli endocarditis: a rare case report. Infect Dis Ther. 2021;10:583-593. doi:10.1007/s40121-020-00377-4

17. Peirano G, Pitout JD. Molecular epidemiology of Escherichia coli producing CTX-M beta-lactamases: the worldwide emergence of clone ST131 O25:H4. Int J Antimicrob Agents. 2010;35:316-321. doi:10.1016/j.ijantimicag.2009.11.003
18. Yano H, Uemura M, Endo S, et al. Molecular characteristics of extended-spectrum $\beta$-lactamases in clinical isolates from Escherichia coli at a Japanese tertiary hospital. PLoS One. 2013;8: e64359. doi:10.1371/journal.pone.0064359

19. Kojima A, Ishii Y, Ishihara K, et al. Extended-spectrum-beta-lactamaseproducing Escherichia coli strains isolated from farm animals from 1999 to 2002: report from the Japanese Veterinary Antimicrobial Resistance Monitoring Program. Antimicrob Agents Chemother. 2005;49:3533-3537. doi:10.1128/AAC.49.8.3533-3537.2005

20. Clermont O, Lavollay M, Vimont S, et al. The CTX-M-15-producing Escherichia coli diffusing clone belongs to a highly virulent B2 phylogenetic subgroup. J Antimicrob Chemother. 2008;61:10241028. doi: $10.1093 / \mathrm{jac} / \mathrm{dkn} 084$

21. Clermont O, Christenson JK, Denamur E, Gordon DM. The Clermont Escherichia coli phylo-typing method revisited: improvement of specificity and detection of new phylo-groups. Environ Microbiol Rep. 2013;5:58-65. doi:10.1111/1758-2229.12019

22. Martinez-Medina M, Mora A, Blanco M, et al. Similarity and divergence among adherent-invasive Escherichia coli and extraintestinal pathogenic E. coli strains. J Clin Microbiol. 2009;47:3968-3979. doi:10.1128/JCM.01484-09

23. Lahlaoui H, Ben Haj Khalifa A, Ben Moussa M. Epidemiology of Enterobacteriaceae producing CTX-M type extended spectrum $\beta$ lactamase (ESBL). Med Mal Infect. 2014;44:400-404. doi:10.1016/ j.medmal.2014.03.010
Infection and Drug Resistance

\section{Publish your work in this journal}

Infection and Drug Resistance is an international, peer-reviewed openaccess journal that focuses on the optimal treatment of infection (bacterial, fungal and viral) and the development and institution of preventive strategies to minimize the development and spread of resistance. The journal is specifically concerned with the epidemiology of

\section{Dovepress}

antibiotic resistance and the mechanisms of resistance development and diffusion in both hospitals and the community. The manuscript management system is completely online and includes a very quick and fair peerreview system, which is all easy to use. Visit http://www.dovepress.com/ testimonials.php to read real quotes from published authors. 